\title{
Howard S. An, Kern Singh: Synopsis of spine surgery
}

\author{
2008, 290 pp., 100 ill. Softcover EUR(D) 54,95 CHF 91,20 Georg Thieme Verlag, \\ Stuttgart, New York, ISBN 13: 978-3-13 143112-7
}

\author{
Pierre H. Kehr • Denis L. Kaech
}

Received: 11 June 2009 / Accepted: 30 June 2009 / Published online: 31 July 2009

(C) Springer-Verlag 2009

Written in "check-list" style, supplemented with examples of imaging, anatomical drawings and summarizing tables, this condensed synopsis is useful for acquiring and repeating the basic knowledge, for preparing a MC or oral examination focusing on spinal pathologies (neurosurgery, orthopedic surgery).

The reader will first find an overwiev of anatomy and surgical approaches, followed by essential points of history taking and physical or brief neurological examination.

Imaging modalities are listed with only a few examples.

The next chapters about biomechanics of the spine and spinal instrumentation is again a condensed check-list with more detailed informations and radiographic examples accompanying anatomical/technical drawings especially of the upper cervical spine. Denis 3 column classification and White \& Panjabi's criteria for lumbar segmental instability are well illustrated. A short section is dedicated to physiology of bone grafts and bone graft substitute, listing some facts in a catalogue style.

The next section II is dedicated to trauma: Glasgow Coma, Frankel impairment and ASIA scales are reproduced, pharmacological agents used in spinal cord injury listed in a table, but not discussed in detail. The following short section about rehabilitation of back and neck pain, impairment and disability evaluation preceeds two partially illustrated chapters about cervical spine trauma and thoraco-lumbar spine fractures.

P. H. Kehr $(\bowtie)$

SOTEST, Marseille, France

e-mail: kehrpier@aol.com

D. L. Kaech

Kantonsspital, Chur, Switzerland
Part II deals with degenerative spinal conditions including a review of the bio-mechanical aspects of disc degeneration, surgical and non-surgical treatment of cervical spine disorders, thoracic spine, lumbar disc disease and lumbar stenosis.

There are some valuable illustrations of referred pain zones, of principles/anatomy of cervical foraminotomy, of surgical anatomy for routine procedures in the lumbar spine. Techniques, advantages and disadvantages of different types of fusion procedures for axial back pain are listed in a last sub-chapter.

Part IV is dedicated to deformity: Lumbar spondylolisthesis. scoliosis and kyphotic spinal deformities in children and adults; Part V to spinal tumors, infections, rheumatoid arthritis, seronegative spondyloarthropathies and pediatric C-Spine disorders. The reader will find some useful tables and illustrative case examples supporting the message of the condensed written text.

At the end of each section 3-10, exceptionally 20 or even 27 references from the late 1980s to 2006 are listed.

In summary, this synopsis can be recommended to junior doctors in training and to physicians involved in the care of patients with spinal problems. It is however not descibing in details "everything one would like to know about spine".

The illustrations in this book should be studied also by rehabilitation specialists, physiotherapists, interested practionners; they may be a help for explaining to many patients what's wrong with their back.

Conflict of interest statement No funds were received in support of this study. 